\title{
Effets d'un aliment dilué par de la luzerne sur les performances et la mortalité de lapines reproductrices
}

\author{
J.Y. JOUGLAR et F. LEBAS *
}

avec la collaboration technique de Béat:ice LOUPIAC, A. LAPANOUSE, A. FALOURD

Chaire de Pathologie Médicale du Bétail et des Animaux de Basse-Cour

Ecole Nationale Vétérinaire, 23, chemin des Capelles, $F 31076$ Toulouse Cedex

*I.N.R.A., Laboratoire de Recherches sur l'Elevage du Lapin

Centre de Recherches de Toulouse, B.P. 27, F 31326 Castanet-Tolosan Cedex

\section{Résumé}

Les auteurs ont distribué à des lapines reproductrices, soit un aliment concentré (Lot $\mathrm{T}$ ), soit le même dilué par 50 p. 100 de luzerne déshydratée (Lot $\mathrm{L}$ ).

Dans un premier essai, les aliments ont été distribués de 5 semaines avant la première saillie jusqu'au sevrage de la première portée, 29 jours après mise bas ; 16 lapines ont reçu l'aliment $\mathrm{L}$ et 13 l'aliment $T$.

Dans la deuxième expérience, 10 lapines multipares par lot n'ont reçu les aliments $L$ ou $T$ que du $28^{\circ}$ jour de leur gestation au sevrage de la portée correspondante. La taille de leur portée a été ajustée après la naissance à 10 lapereaux en moyenne par adoption ou retrait maximum d'un lapereau.

La distribution de l'aliment $\mathrm{L}$ a entraîné la mort de deux lapines sur 10 dans la deuxième expérience, mais aucune dans la première. Dans les deux essais, les lapines recevant l'aliment $\mathrm{L}$ ont $\mathrm{cu}$ une consommation moyenne supérieure d'environ $10 \mathrm{p} .100$ par rapport à celle des lapines $T$. La surconsommation a été particulièrement marquée durant le début de lactation.

En $3^{\text {c }}$ semaine de lactation, les lapines recevant l'aliment $\mathbf{L}$ ont une perte de poids significativement plus forte que les lapines témoins : - $208 \mathrm{~g} \mathrm{vs}-66 \mathrm{~g}$.

Les réserves corporelles au moment du sevrage ne sont pas différentes chez les lapines n'ayant reçu les aliments expérimentaux que durant la lactation. Elles sont réduites lorsque l'aliment $\mathrm{L}$ a été distribué avant la première saillie (Essai $\mathrm{n}^{\circ} 1$ ).

La distribution de l'aliment $\mathrm{L}$ s'est également traduite par une réduction du poids total des portées au sevrage. A l'inverse, la mortalité des lapereaux entre 0 et 29 jours n'a été que de 8 p. 100 pour l'ensemble des lapines L, contre 14,4 p. 100 pour l'ensemble des lapines recevant l'aliment témoin. De la discussion, il ressort que l'excès de calcium de l'aliment $\mathbf{L}$ et sa déficience en énergie digestible sont très probablement responsables d'une fraction des phénomènes observés.

Mots clés : Lapine, reproduction, mortalité, luzerne, équilibre alimentaire. 


\section{Introduction}

Au cours d'une expérimentation conduite au Laboratoire de Recherches sur l'Elevage du Lapin, une mortalité brutale très élevée $(65 \mathrm{p} .100)$ a été observée pendant la 3 " semaine de lactation chez les lapines primipares allaitantes, recevant un aliment expérimental constitué pour moitié de luzerne déshydratée et pour moitié d'un aliment équilibré répondant aux recommandations nutritionnelles (LANG, 1981). L'objectif de l'expérience était d'élever ces animaux sur un régime unique avant de les étudier, eux et leurs descendants, en les alimentant, soit avec un aliment équilibré, soit uniquement avec de la luzerne (Jouglar, 1983).

L'analyse du problème nous a conduit à émettre l'hypothèse que la composition de l'aliment utilisé était responsable des mortalités. Nous avons donc mis en place deux expérimentations dans le but de vérifier cette hypothèse. Dans la première, l'aliment expérimental a été distribué pendant tout le premier cycle de reproduction, tandis que dans la deuxième, l'aliment n'a été distribué que pendant la lactation à des lapines multipares.

\section{TABleau 1}

Composition des aliments expérimentaux.

Composition of the experimental diets.

\begin{tabular}{|c|c|c|c|}
\hline \multicolumn{2}{|r|}{ Type d'aliment - Type of diet } & $\mathrm{L}$ & $\mathrm{T}$ \\
\hline \multicolumn{2}{|c|}{ Composants - Components } & $\%$ & \\
\hline \multicolumn{2}{|c|}{ Luzerne - Lucerne } & 65,5 & 31 \\
\hline \multicolumn{2}{|c|}{ Blé - Wheat .... } & 12 & 24 \\
\hline \multicolumn{2}{|c|}{ Avoine-Oats } & 7.5 & 15 \\
\hline \multicolumn{2}{|c|}{ Tourteau de soja - Soyabean meal } & 45 & 9 \\
\hline \multicolumn{2}{|r|}{ Tourteau de tournesol - Sunflower meal ............. } & 40 & 8 \\
\hline \multicolumn{2}{|c|}{ Pulpes de betteraves - Dried beet pulp } & 4,5 & 9 \\
\hline \multicolumn{2}{|r|}{ CMV 853 - Minerals and vitamins $(1) \ldots \ldots \ldots \ldots$} & 2,0 & 4 \\
\hline \multicolumn{2}{|r|}{$\begin{array}{l}\text { Composition analytique }- \text { Analytical composition }(\%) \\
\text { Teneur en MS - Dry matter }(\%) \ldots \ldots \ldots \ldots \ldots \ldots\end{array}$} & 88,4 & 88,7 \\
\hline \multirow{2}{*}{$\underset{0}{\vdots}$} & Matière azotée totale $(\mathrm{N} \times 6,25)$ - Crude protein & 17,1 & 17,8 \\
\hline & Cellulose brute (Weende) - Crude fibre ......... & 25,7 & 17,4 \\
\hline 50 & Matières minérales totales - Minerals & 102 & 10 \\
\hline \multirow{2}{*}{$\sum_{i 0}^{\infty}$} & Calcium - Calcium & 1,85 & 1,57 \\
\hline & Phosphore total - Phosphorus .. & 0,47 & 0,73 \\
\hline \multicolumn{2}{|r|}{$\begin{array}{l}\text { Energie digestible } \mathrm{kcal} / \mathrm{kg} \text { MS (2) } \ldots \ldots \ldots \ldots \ldots \ldots \ldots \\
\text { Digestible energy } \mathrm{kcal} / \mathrm{kg} \text { DM }\end{array}$} & 2575 & 2775 \\
\hline
\end{tabular}

(1) Colin, Arkhurst \& Lebas (1973).

(2) Calcul après mesure in vivo pour la luzerne et utilisation des valeurs tabulaires I.N.R.A. (1984) pour les autres matières premières.

Calculation after measurement in vivo for lucerne and utilization of I.N.R.A. tables for the other feeds. 


\section{Matériel et méthodes}

\section{A. Conditions générales}

Au total, 49 lapines de race néozélandaise blanches ont été utilisées. Elles ont été logées dans des cages individuelles de reproduction placées dans un bâtiment à ventilation contrôlée et chauffé à $19 \pm 1^{\circ} \mathrm{C}$ en période hivernale.

La composition de l'aliment témoin ( $T$ ) et de l'aliment expérimental (L) est fournie au tableau 1. Ils ont été fabriqués à partir du même stock de matières premières que l'aliment impliqué dans l'observation initiale. L'aliment témoin a été formulé pour satisfaire aux recommandations nutritionnelles. L'aliment expérimental, identique à celui utilisé dans l'observation préalable (Jouglar, 1983), contenait 65,5 p. 100 de luzerne déshydratée contre 31 p. 100 dans l'aliment témoin. Cet aliment expérimental correspond au remplacement de la moitié des matières premières et additifs (minéraux, vitamines) de l'aliment témoin par de la luzerne déshydratée.

Les deux aliments sont pratiquement isoazotés, mais l'aliment expérimental est plus riche en cellulose et en calcium et plus pauvre en phosphore et en énergie digestible.

\section{B. La première expérimentation : Femelles primipares}

Le protocole de cette expérimentation reprend scrupuleusement le protocole de l'observation initiale en respectant toutes les conditions d'élevage imposées. Deux lots de jeunes femelles, âgées de 100 jours, pesant en moyenne $3200 \mathrm{~g}$ sont constitués : le lot $\mathrm{L}$ recevant l'aliment expérimental comprend 16 animaux, le lot $\mathrm{T}$, témoin, regroupe 13 animaux. La chronologie des événements de la première expérience est représentée sur la figure 1.

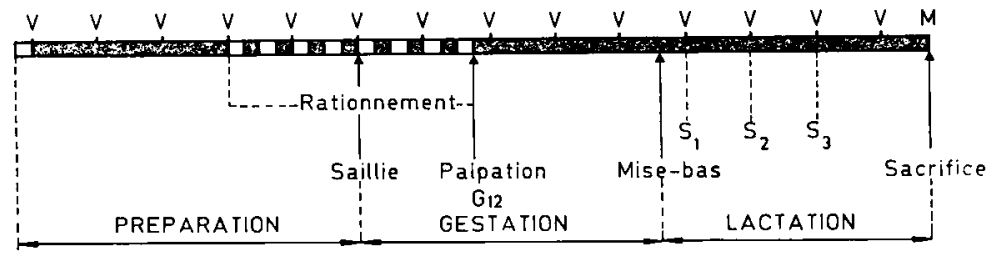

FIG. 1

Protocole expérimental de la première expérience. Experimental design of the first experiment.

V : Vendredi.

Friday.

S1 : Saillie post partum.

Post partum mating.

S2 : Saillie si refus en S1.

Mating if refusal in $S 1$.

S3 : Saillie si S1 inféconde.

Mating if unfertilized mating in S1. 
Après une période d'alimentation ad libitum de 3 semaines, les lapines subissent un rationnement à 150 grammes par jour, quel que soit l'aliment, jusqu'au moment où leur gestation est confirmée par palpation 12 jours après la saillie.

La première présentation au mâle a lieu après deux semaines de rationnement. La période de restriction alimentaire dure donc 27 jours pour les femelles qui acceptent le mâle dès la première fois. A partir de 12 jours de gestation et jusqu'à la fin de la lactation, les animaux sont de nouveau alimentés à volonté. Les femelles sont présentées au mâle 3 à 4 jours après la mise bas. En cas de refus en S1, une deuxième tentative a lieu une semaine plus tard (S2). En cas de saillie ne donnant pas lieu à gestation (contrôle 12 jours post coïtum), les lapines sont représentées au mâle deux semaines après la première tentative (S3).

\section{La deuxième expérimentation : Femelles multipares}

$\mathrm{Au} 28^{\circ}$ jour de la gestation, des lapines ayant déjà effectué au moins une mise bas ont été réparties équitablement entre les deux lots correspondant à la distribution à volonté de l'un des deux aliments expérimentaux.

Après la mise bas, n'ont été retenues que les lapines ayant donné naissance au moins à 7 lapereaux vivants, de façon que les effectifs de portées à JO soient équivalents pour les deux groupes (10 lapereaux vivants). Au total, 10 lapines ont été retenues pour chacun des deux lots. La prise en compte uniquement des lapines ayant plus de 7 lapereaux nous est apparue intéressante pour tester l'aliment expérimental sur des femelles allaitant des portées nombreuses, ayant donc des besoins élevés. Ces femelles n'ont pas été présentées au mâle après la mise bas, de façon à apprécier l'effet des aliments sur les femelles uniquement allaitantes.

\section{Contrôles expérimentaux}

Dans la première expérimentation, les lapines ont été pesées à l'âge de 100 jours, au début du rationnement, à la première présentation au mâle, aux $7^{\mathrm{e}}, 14^{\mathrm{e}}, 21^{\mathrm{e}}$ et $28^{\mathrm{e}}$ jours de gestation. Dans le cas des deux expérimentations, les lapines ont été pesées le jour de la mise bas, puis 3 fois par semaine. La consommation alimentaire a été contrôlée 3 fois par semaine à partir du $12^{\circ}$ jour de gestation jusqu'aux $29^{\circ}-30^{\circ}$ jours après mise bas, date du sacrifice des femelles. Les lapereaux ont été pesés individuellement à la mise bas, puis une fois par semaine jusqu'au sevrage (29-30 jours).

Au moment du sacrifice des femelles, ont été contrôlés, le poids de la carcasse commerciale (après $24 \mathrm{~h}$ à $+4^{\circ} \mathrm{C}$, sans manchons), celui de la peau, du tube digestif plein puis vide, le poids du foie, des deux reins et celui d'une cuisse. Le poids des os de la cuisse a été également déterminé. Sur l'ensemble osseux (fémur + coxal), les teneurs en calcium (méthode par spectrométrie d'absorption atomique) et en phosphore (méthode colorimétrique au vanadate) ont été déterminées après minéralisation deux heures à $500^{\circ} \mathrm{C}$. Au moment du sacrifice, la présence de corps cétoniques a été recherchée dans l'urine vésicale des lapines pour tester l'hypothèse d'une cétose associée à la distribution de l'aliment expérimental, par analogie au phénomène observé sur les vaches laitières en déficit énergétique. La méthode utilisée était celle des bandelettes permettant de déceler $0,1 \mathrm{~g} / 1$ d'acide acéto-acétique ou $0,7 \mathrm{~g} / 1 \mathrm{~d}$ 'acétone $\left(^{*}\right)$.

${ }^{(*)}$ Ketur-Test $\mathbf{R}$ commercialisé par les Ets Boehringer Mannheim (Allemagne Fédérale). 
Enfin, des examens histologiques ont été effectués sur le foie et les reins par le Laboratoire de la Chaire d'Anatomie Pathologie de l'Ecole Nationale Vétérinaire de Toulouse, de manière à rechercher d'éventuelles lésions microscopiques majeures sur ces organes.

\section{E. Analyse mathématique des résultats}

Les données numériques ont été étudiées par analyse de variance avec ou sans covariable grâce au programme $C \mathrm{~N} N \mathrm{~N}$ mis en œuvre sur l'ordinateur MITRA 15 du laboratoire de Biométrie du C.R. I.N.R.A. de Toulouse. Ce programme permet de traiter un ou plusieurs facteurs contrôlés sur des populations d'effectifs déséquilibrés (Bachacou, Masson \& Millier, 1981). Les comparaisons de taux et de proportion ont été faites par test de $\chi^{2}$ de Pearson.

\section{Résultats}

\section{A. Déroulement des expérimentations}

Deux lapines du lot $\mathrm{L}$ sont mortes dans l'expérience 2 , 1'une au $22^{\circ}$ jour et l'autre au $25^{\circ}$ après la mise bas; par contre, aucune autre perte de femelle n'a été constatée ni pour le lot témoin de cette expérience, ni pour aucun des deux lots de l'expérience 1. Pour ces deux lapines, le tableau clinique a été analogue à celui décrit dans l'observation initiale. Leur poids à la mise bas était très proche de la moyenne du lot. Après une prise de poids très rapide en première semaine de lactation $(+300$ à $400 \mathrm{~g}$ contre $+180 \mathrm{~g}$ pour les autres femelles), nous avons constaté une perte de poids très importante à partir du $14^{\circ}$ jour de lactation. Ces deux femelles ont néanmoins allaité 11 et 9 lapereaux jusqu'au moment de leur mort. Pour l'analyse ultérieure des performances, seules les lapines vivantes en fin d'essai ont été retenues.

Dans la première expérimentation, l'aliment $\mathrm{L}$ n'a eu aucune incidence sur la réussite des premiers accouplements, toutes les femelles ayant été gravides dès la première saillie, dans les deux lots. Par contre, après la première mise bas, la remise en gestation semble plus difficile pour le lot $\mathrm{L}$ que pour le lot $\mathrm{T}$ (tabl. 2), mais les écarts ne sont pas statistiquement significatifs.

\section{B. Consommation alimentaire et poids des femelles}

Dans la première expérience, dès que la distribution ad libitum est rétablie, l'ingestion d'aliment $L$ est significativement supérieure à celle de l'aliment témoin : $+30,8$ p. 100 entre G 12 et la mise bas (fig. 2). Dans les 2 jours qui suivent la mise bas, la reprise de consommation est beaucoup plus marquée dans le lot $\mathrm{T}(+162 \mathrm{p} .100$ par rapport aux 2 jours précédant la mise bas) que dans le lot $L(+22$ p. 100). Dans la semaine qui suit, la consommation du lot $\mathrm{L}$ redevient supérieure à celle du lot $\mathrm{T}$ : $+13,1$ p. 100. Par la suite, les consommations sont équivalentes jusqu'au sevrage.

Un comportement alimentaire analogue des lapines du lot $\mathrm{L}$ est observé dans la deuxième expérience (fig. 3), bien que les femelles des deux lots aient eu la même alimentation jusqu'au $28^{\circ}$ jour de gestation. La surconsommation d'aliment $L$ se prolonge ici jusqu'au $16^{\circ}$ jour de lactation. 


\section{TABleau 2}

Présentation au mâle et fécondation des lapines après la première mise bas dans l'expérience I. Mating acceptance and fertilization of doe rabbits after the first parturition in experiment 1.

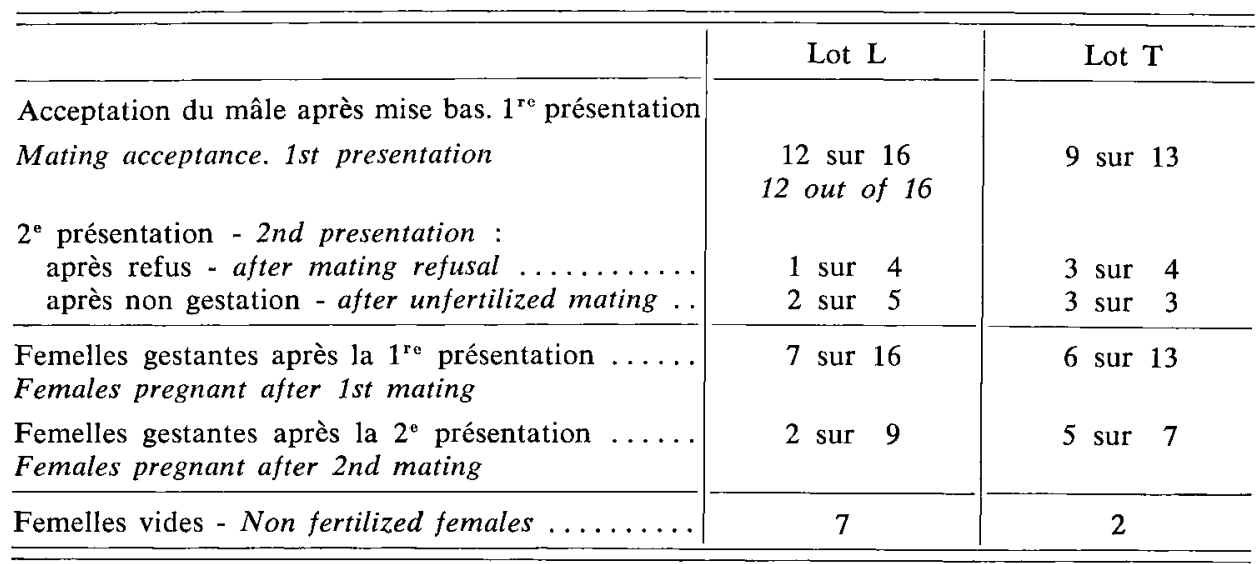

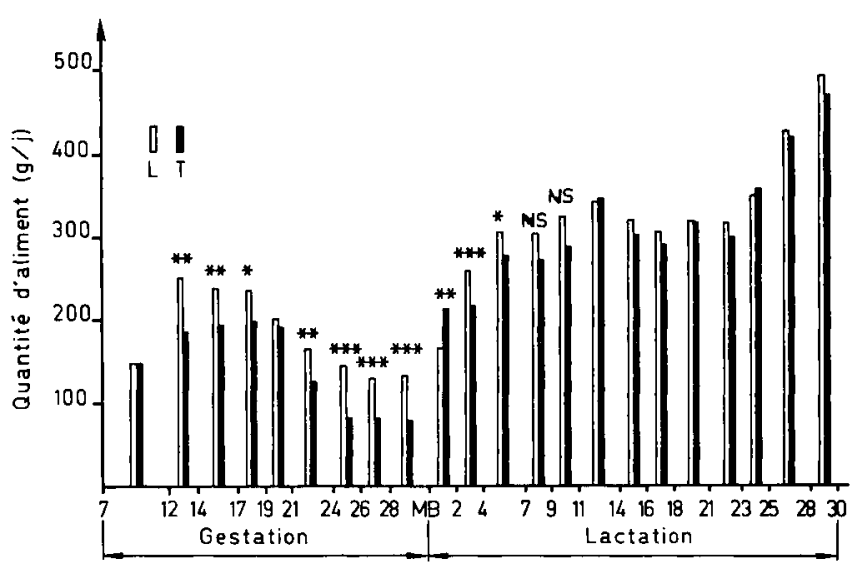

FIG. 2

Evolution de la consommation alimentaire moyenne journalière par lapine dans l'expérience 1 (lapine + portée à partir de 21 jours post partum).

Experiment 1 : variations in the mean daily food intake of the doe and its litter (21 days post partum).

* $\mathrm{P}<0,10 ; * * \mathrm{P}<0,05 ; * * * \mathrm{P}<0,01$.

L'évolution du poids des lapines entre la mise en lot et la fin de la première expérience est décrite sur la figure 4. La distribution des deux aliments à volonté pendant 3 semaines après la mise en lot suivie d'une restriction alimentaire (150 $\mathrm{g}$ 
par jour) se traduit, au moment de la saillie, par une réduction significative de $400 \mathrm{~g}$ du poids des lapines $\mathrm{L}$ par rapport aux témoins. Cet écart est ensuite maintenu pendant l'ensemble du cycle de production.

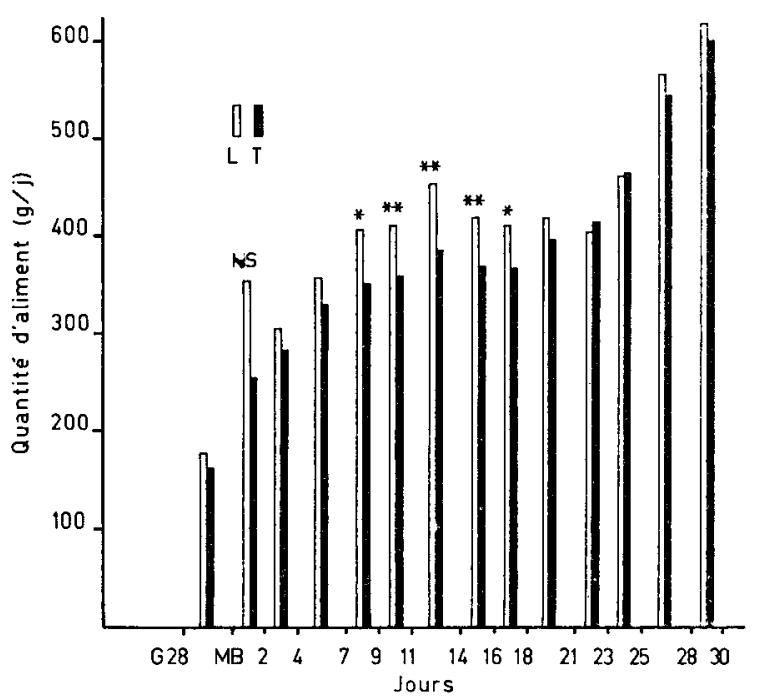

FIG. 3

Evolution de la consommation alimentaire moyenne iournalière par lapine dans l'expérience 2 (lapine + portée à partir de 21 jours post partum).

Experiment 2 : Variation in the mean daily food intake of the doe (and its litter after the 21th days post partum).

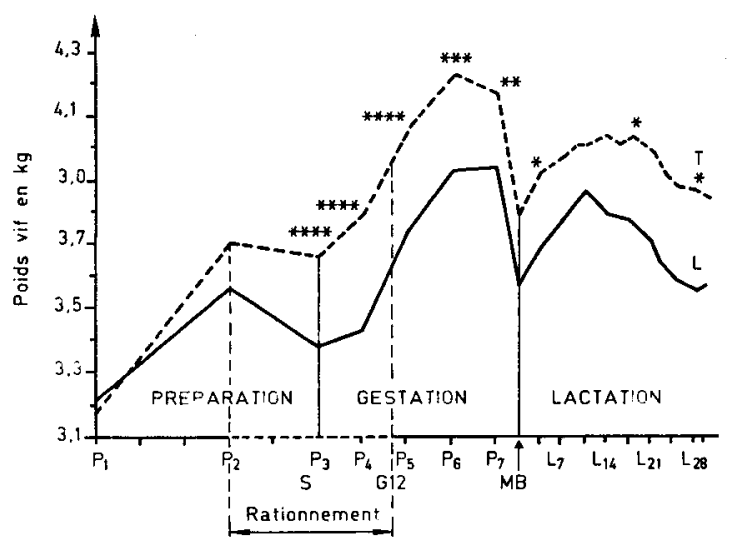

FIG. 4

Evolution du poids vif des lapines dans l'expérience 1.

Variation in the live weight of does during the 1st experiment.

* $\mathrm{P}<0,10 ; * * \mathrm{P}<0,05 ; * * * \mathrm{P}<0,01 ; * * * * \mathrm{P}<0,001$. 
Nous avons cherché à savoir si l'état éventuel de gestation est susceptible de modifier l'évolution du poids vif des lapines allaitantes. Pour les lapines du lot $\mathrm{L}$ l'évolution du poids vif est comparable chez celles fécondées 3-4 jours après mise bas $(n=6)$ et chez les autres $(n=7)$. Par contre, nous avons constaté une baisse marquée du poids vif, de $200 \mathrm{~g}$, entre $\mathrm{L} 11$ et $\mathrm{L} 21$ pour les 9 lapines du lot $\mathrm{L}$ uniquement allaitantes, alors que les 7 lapines témoins non gestantes perdent seulement $50 \mathrm{~g}$ pendant cette période. Cette différence d'évolution est significative. Pour les 7 lapines du lot $\mathrm{L}$ gestantes dès la première saillie post partum, cette forte chute de poids n'est pas constatée.

Au moment de leur saillie, les lapines de la deuxième expérience avaient des poids comparables dans les deux groupes : lot L $3916 \pm 217 \mathrm{~g}$; lot $\mathrm{T} 4022 \pm 128 \mathrm{~g}$. L'écart numérique observé à la mise bas entre les poids vifs des 2 lots de femelles n'est pas significatif. Il correspond à une légère accentuation de la perte de poids en fin de gestation pour le lot L, mais cette tendance n'est pas significative.

L'évolution du poids après la mise bas est similaire à celle observée dans l'expérience 1.

Nous retrouvons une chute de poids significativement plus forte entre 16 et 23 jours de lactation pour le lot L (-208 g) que le lot T (-66 g) (fig. 5).

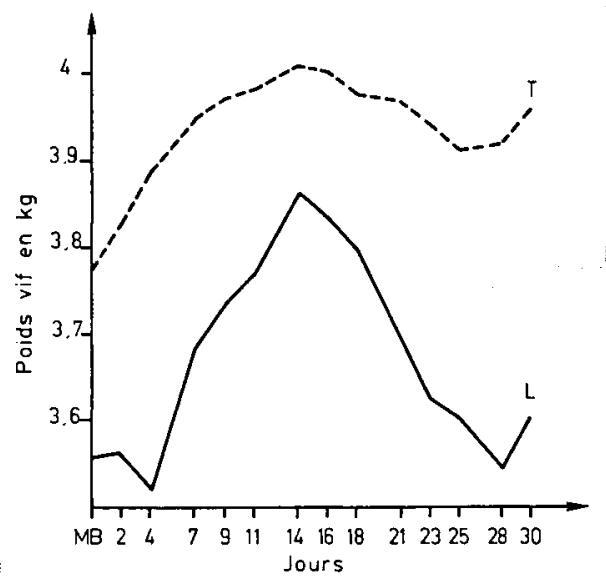

FIG. 5

Evolution du poids vif des lapines dans l'expérience 2.

Variation in the live weight of does during the 2 nd experiment.

\section{Caractéristiques des lapines en fin de lactation}

Pour aucune des lapines de chacune des deux expériences nous n'avons décelé de corps cétoniques dans l'urine vésicale.

Nous avons représenté sur la figure 6 les variations (p. 100) par rapport aux témoins des valeurs moyennes des variables mesurées lors du sacrifice des lapines $\mathrm{L}$ 
(après analyse sans covariable, puis analyse de covariance avec pour covariable le poids vif vide) :

- pour l'expérience 1 , le poids vif vide est défini comme le poids vif à l'abattage réduit du contenu digestif et du contenu de l'utérus gravide ;

- pour l'expérience 2 , le poids vif à l'abattage réduit du contenu digestif (lapines nécessairement non gestantes).

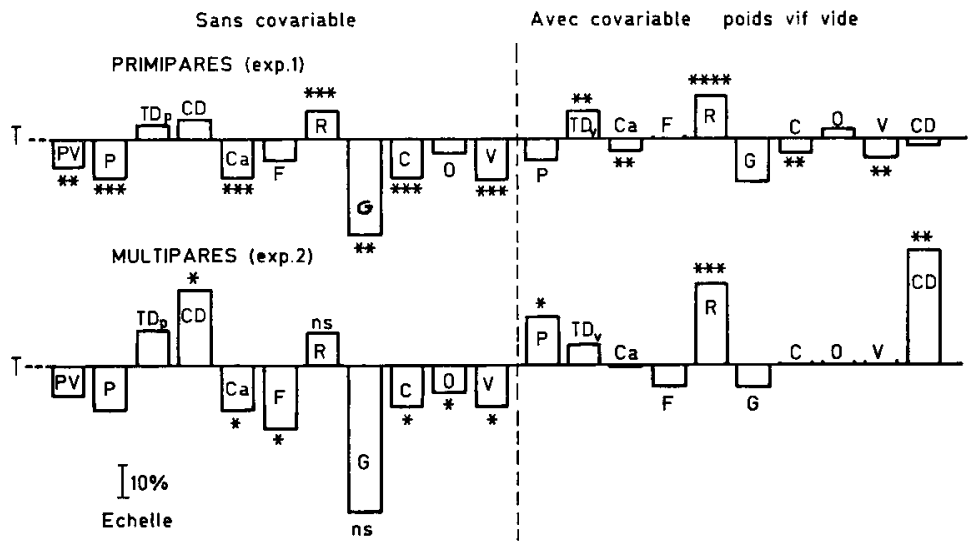

FIG. 6

Effets du régime alimentaire $L$ sur l'état corporel en fin de lactation, pour les deux expériences : p. 100 valeur aliment $T$.

Effect of diet $L$ on the body composition of females in late lactation for both. experiments : p. 100 diet $T$.

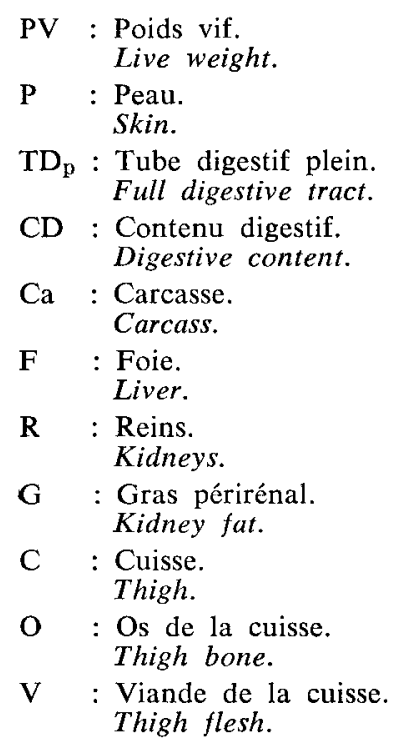

$\mathrm{TD}_{\mathrm{r}}$ : Tube digestif vide.

Empty digestive tract. 
L'analyse sans covariable permet de mettre en évidence des résultats similaires pour les deux expériences, démontrant ainsi l'effet défavorable de l'aliment L sur l'état corporel des femelles en lactation. Les carcasses du lot $\mathrm{L}$ sont plus légères $(250$ à $300 \mathrm{~g})$ que celles des témoins. Ce résultat est lié directement aux différences de poids vif existant entre les deux groupes. En effet, pour l'expérience 2, l'analyse de covariance (poids vif vide moyen de $3300 \mathrm{~g}$ ) montre un poids de carcasse identique pour les deux lots. Par contre, pour l'expérience 1, l'analyse de covariance révèle comme l'analyse sans covariable que les carcasses du lot $\mathrm{L}$ sont plus légères et moins musclées que celles des témoins. Les réserves de graisses, représentées par le gras périrénal, sont également diminuées sur les lapines L. Par contre, les réserves minérales au niveau du fémur et du coxal ne semblent pas modifiées par l'alimentation (tabl. 3).

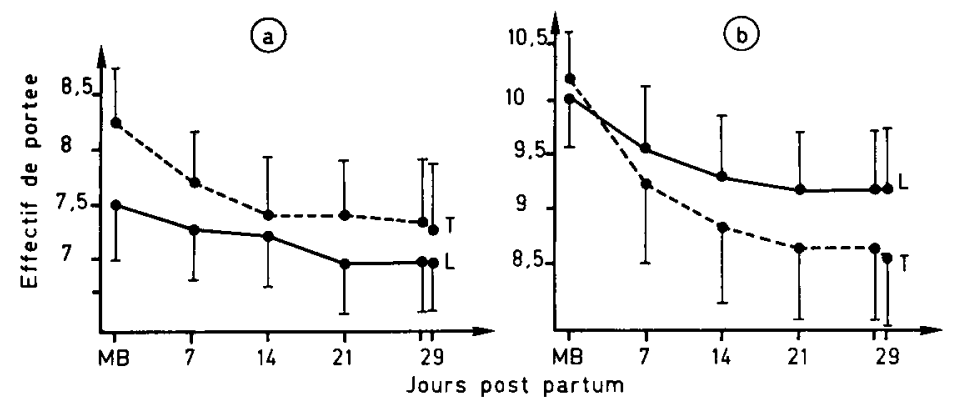

FIG. 7

Evolution de la taille des portées entre la naissance et le sevrage dans l'expérience 1 (a) et dans l'expérience 2 (b).

Variation in the litter size between birth and weaning in experiment 1 (a) and 2 (b).

Nous avons noté également une augmentation du poids des reins pour les lapines du lot L, mais l'observation des coupes histologiques n'a permis de déceler aucune lésion rénale, ni d'ailleurs aucune lésion hépatique.

\section{Viabilité et croissance des lapereaux}

Dans l'expérience 1, nous constatons dans le lot $\mathrm{L}$ une réduction non significative de la taille de la portée à la naissance : 7,5 nés vivants vs 8,2 pour le lot $\mathrm{T}$. Dans la deuxième expérience, les effectifs à la naissance sont plus élevés et identiques dans deux lots en raison même du protocole adopté : 10,0 nés vivants dans le lot $\mathrm{L}$ contre 10,2 dans le lot $T$.

Pour les deux expériences prises l'une après l'autre, les analyses statistiques ne permettent de déceler de différence significative d'effectif pour aucun des stades de mesure (fig. 8).

Mais, si nous cumulons les résultats des deux expériences, le test $\chi^{2}$ démontre que les lapereaux du lot $\mathrm{L}$ sont moins affectés par les mortalités avant le sevrage que 


\section{TABLEAU 3}

Composition minérale des os de la cuisse dans les deux expériences Moyennes (x) en p. 100 de la matière sèche et coefficient de variation (CV \%).

Aucune différence significative entre lots.

Mineral composition of hind leg bones in the 2 experiments.

Mean $(\bar{x})$ expressed as percent of the dry matter, and coefficient of variation (CV \%). Variations between groups are non significant.

\begin{tabular}{|c|c|c|c|c|c|c|c|c|}
\hline & \multicolumn{4}{|c|}{ Expérience 1 - Experiment 1} & \multicolumn{4}{|c|}{ Expérience 2 - Experiment 2} \\
\hline & \multicolumn{2}{|c|}{ Lot $\mathrm{T}$} & \multicolumn{2}{|c|}{ Lot $\mathrm{T}$} & \multicolumn{2}{|c|}{ Lot $\mathbf{L}$} & \multicolumn{2}{|c|}{ Lot $\mathbf{L}$} \\
\hline & $\overline{\mathbf{x}}$ & $\mathrm{CV} \%$ & $\overline{\mathbf{x}}$ & $\mathrm{CV} \%$ & $\overrightarrow{\mathbf{x}}$ & $\mathrm{CV} \%$ & $\overline{\mathbf{x}}$ & $\mathrm{CV} \%$ \\
\hline Fémur - Femur & & & & & & & & \\
\hline $\begin{array}{l}\text { Minéraux totaux } \ldots \ldots \\
\text { Minerals }\end{array}$ & 52,2 & 2,4 & 54,7 & 6,4 & 52,1 & 4,0 & 52,0 & 4,4 \\
\hline Calcium ... & 19,1 & 2,1 & 20,3 & 6,0 & 19,2 & 4,4 & 19,1 & 4,6 \\
\hline Phosphore-Phosphorus & 9,1 & 3,0 & 9,5 & 6,3 & 9,0 & 4,1 & 9,0 & 4,7 \\
\hline Coxal - Hipbone & & & & & & & & \\
\hline $\begin{array}{l}\text { Minéraux totaux } \ldots \\
\text { Minerals }\end{array}$ & 51,7 & 5,7 & 53,1 & 9,3 & 51,7 & 6,1 & 51,4 & 5,7 \\
\hline Calcium $\ldots \ldots \ldots$ & 18,8 & 5,5 & 19,1 & 8,5 & 18,4 & 6,3 & 18,4 & 6,1 \\
\hline Phosphore - Phosphorus & 9,0 & 6,0 & 9,2 & 9,0 & 8,7 & 5,8 & 8,7 & 6,0 \\
\hline
\end{tabular}
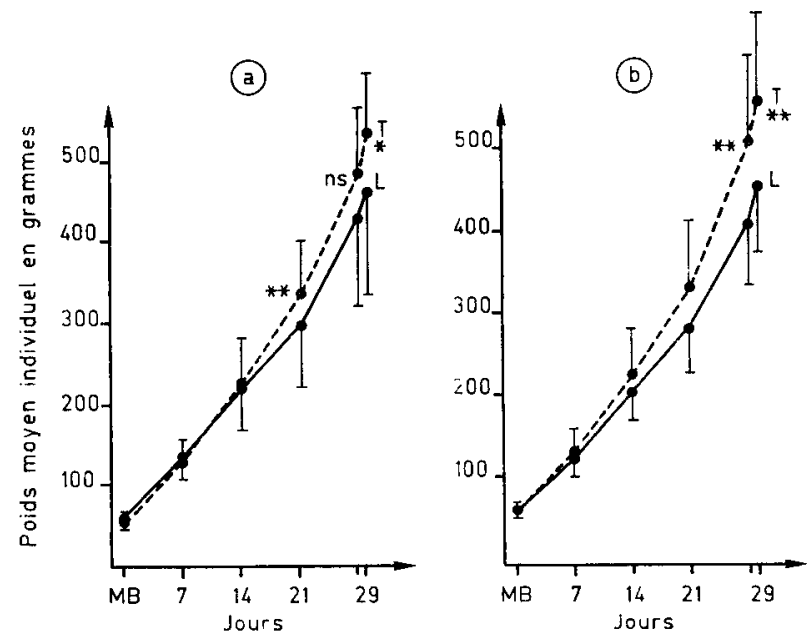

FIG. 8 a

Evolution du poids vif individuel moyen des lapereaux entre la naissance et le sevrage dans l'expérience 1.

$V$ ariation in the live weight of young rabbits between birth and weaning during experiment $I$.

Fig. 8 b

Evolution du poids vif individuel moyen des lapereaux entre la naissance et le sevrage dans la deuxième expérience.

Variation in the live weight of young rabbits between birth and weaning during experiment 2. 
les témoins; en effet, la mortalité entre 0 et 28 jours n'est que de 8,0 p. 100 pour les deux lots L., alors qu'elle atteint 14,4 p. 100 pour l'ensemble des deux lots $\mathrm{T}(\mathrm{P}<0,05)$. Au plan global, la combinaison des effectifs et des poids individuels conduit à un poids total de portée au sevrage inférieur pour les lapins des deux lots $\mathrm{L}$ par rapport à ceux des deux lots $T$ (tabl. 4). Cependant, la différence n'est significative que dans le cas de la première expérience.

\section{TABLEAU 4}

Poids moyen des portées au sevrage.

Moyenne $(\bar{x})$ exprimée en grammes et coefficient de variation (CV \%).

Mean weight of litters at weaning.

Mean $(\bar{x})$ as grammes and coefficient of variation (CV \%).

\begin{tabular}{|c|c|c|c|c|c|}
\hline & \multicolumn{2}{|c|}{ Lot $\mathrm{L}$} & \multicolumn{2}{|c|}{ Lot $\mathrm{T}$} & \multirow{2}{*}{$\begin{array}{l}\text { Signification } \\
\text { statistique }\end{array}$} \\
\hline & $\bar{x}$ & $\mathrm{CV} \%$ & $\overline{\mathrm{x}}$ & $\mathrm{CV} \%$ & \\
\hline $\begin{array}{l}\text { Expérience } 1 \\
\text { Experiment } 1\end{array}$ & 3071 & 20,9 & 3759 & 22,4 & * \\
\hline $\begin{array}{l}\text { Expérience } 2 . \\
\text { Experiment } 2\end{array}$ & 3945 & 10,1 & 4532 & 21,1 & NS \\
\hline
\end{tabular}

NS : Non significatif - Non significant; * : $\mathbf{P}<0,05$.

\section{Discussion}

La distribution de l'aliment $\mathbf{L}$ pauvre en énergie se traduit par une consommation alimentaire supérieure aussi bien en gestation qu'en début de lactation. On peut considérer qu'il s'agit d'une réaction classique à la dilution de l'énergie alimentaire déjà observée tant chez l'animal en croissance (BombeKe et al., 1978) que chez la lapine reproductrice (LEBAS, 1977).

Toutefois, nous devons remarquer qu'au cours de la $3^{\text {e }}$ semaine, au moment de la plus forte production laitière (LEBAS, 1968), les lapines des lots $\mathrm{L}$ ne consomment pas plus que celles des lots témoins. Ce défaut d'ajustement est probablement lié spécifiquement à la composition de l'aliment $\mathrm{L}$ sans relation avec sa concentration énergétique puisque les lapines recevant un aliment équilibré peuvent adapter leur consommation alimentaire à leur production laitière (LEBAS, 1987).

Un déséquilibre de l'apport protéique lié à la dilution par la luzerne peut être envisagé également, mais aucun acide aminé particulier ne peut être mis en cause puisque les besoins en acides aminés des lapines allaitantes ne sont pas connus (LANG, 1981). Par ailleurs, les différences de niveau d'ingestion ne peuvent être reliées aux écarts de concentration phosphocalcique des aliments puisqu'une variation spécifique de ces teneurs n'entraîne aucune modification de la consommation alimentaire (LEBAS \& Jouglar, 1984). 
Au cours de la troisième semaine de lactation, nous avons noté une chute marquée $\mathrm{du}$ poids vif pour les lapines du lot $\mathrm{L}$ dans les deux expériences. Une semaine plus tard, au moment du sevrage, nous n'observons sur les lapines multipares aucun effet significatif de l'aliment sur la composition corporelle. En conséquence, la relative fonte musculaire et la baisse des réserves adipeuses observées chez les lapines primipares du lot $\mathrm{L}$ par rapport aux témoins ne sont pas liées à une mobilisation éventuellement plus intense des réserves pendant la période d'allaitement. Il est probable que l'effet observé n'est que la conséquence d'une différence de composition corporelle effectivement due à l'aliment mais établie avant le début de la lactation. Le protocole expérimental utilisé ne permet pas de tester cette hypothèse.

L'expérimentation avait été motivée au départ par une tentative d'analyse et de reproduction de mortalité sur des lapines reproductrices. Celle-ci n'a pas été retrouvée dans l'expérience 1 dont le protocole était identique à celui appliqué lors de l'observation initiale. Par contre, dans l'expérience 2, 2 lapines particulièrement prolifiques sont mortes dans des conditions similaires à celles de l'observation initiale (Jouglar, 1983). En conséquence, si l'aliment était bien impliqué dans la mortalité, d'autres facteurs qui n'ont pu être identifiés ont dû intervenir.

Dans l'expérience 1 , la distribution de l'aliment $L$ tend à réduire la taille de la portée à la naissance. Ceci peut être rapproché de l'effet négatif sur la prolificité d'une restriction alimentaire globale précédant la saillie (COUDERT \& LEBAS, 1985).

Un des phénomènes remarquables sur l'ensemble des deux expériences est l'amélioration de viabilité des lapereaux des lots $\mathrm{L}$ entre naissance et sevrage, alors même que leur poids individuel est réduit.

Cette situation ne peut être expliquée ni par les différences de concentration énergétique (LEBAS, 1977), ni par un déséquilibre d'apport phosphocalcique (LeBAS \& JOUGLAR, 1984).

Il est possible que la composition du lait, en particulier celle de ses triglycérides, ait été modifiée dans un sens favorable à la viabilité (Perret, Dorier \& Bacoues, 1977).

Dans les deux expériences nous constatons une réduction du poids moyen des lapereaux du lot $\mathrm{L}$ par rapport aux témoins et corrélativement une réduction du poids total de la portée au sevrage. Une telle réduction globale avait déjà été observée par Lebas \& Jouglar (1984) avec une ingestion excessive de calcium chez les lapines allaitantes : $1,54 \mathrm{vs} 1,00 \mathrm{~g} / \mathrm{kg}$ de poids vif par jour, soit $+41 \mathrm{p} .100$. Effectivement, les lapines du Lot $\mathrm{L}$, en $3^{\circ}$ semaine de lactation, ingèrent +31 p. 100 de calcium par rapport aux témoins dans la première expérience et +27 p. 100 dans la deuxième.

\section{Conclusion}

En conclusion de ces expériences, nous devons constater que l'aliment incriminé dans l'observation initiale perturbe bien les lapines allaitantes. Mais, si nous avons reproduit le type d'accident observé initialement pendant la lactation du point de vue clinique et chronologique, nous ne l'avons pas reproduit avec la même ampleur. 
Au-delà de cette constatation, la distribution d'un aliment dilué par de la luzerne entraîne des modifications sensibles des performances des lapines reproductrices, essentiellement durant la période d'allaitement. En effet, la distribution uniquement pendant cette période entraîne des conséquences très comparables à celles d'une distribution commencée plus tôt, dès avant la première saillie des jeunes lapines. Il paraît probable que l'excès de calcium et surtout la déficience en énergie de l'aliment riche en luzerne soient responsables des perturbations observées, mais ces deux caractéristiques de l'aliment ne peuvent à elles seules expliquer la totalité des phénomènes observés.

Reçu en octobre 1984.

Accepté en mai 1986.

\section{Remerciements}

Les auteurs tiennent à remercier M. le Professeur CaBanie qui a interprété les coupes histologiques et M. Auriac, Maître-Assistant à Ia Faculté de Pharmacie de Toulouse qui a permis l'étude de la minéralisation des os.

\section{Summary \\ Effect of feeding dehydrated lucerne diluted diet to breeding doe rabbits on performance and mortality}

Breeding doe rabbits were fed either concentrate (group $\mathrm{T}$ ) or the same diet admixed with 50 p. 100 dehydrated lucerne (group L).

In the first experiment, diets were offered from 5 weeks before the first mating until weaning of the first litter, 29 days after littering. Sixteen doe rabbits received diet $L$ and thirteen diet $T$.

In the second experiment, 10 multiparous does per group were fed diet $\mathrm{L}$ or $\mathrm{T}$ only from day 28 of pregnancy until weaning of the corresponding litter. The litter size was adjusted to 10 young rabbits by adding or withdrawing one young.

Two doe rabbits out of ten fed diet $\mathrm{L}$ died in the second experiment, but none in the first one. In both trials, the feed intake of does fed diet $\mathrm{L}$ was about $10 \mathrm{p}$. 100 higher than that of does fed diet $T$. Overfeeding was particularly marked in early lactation.

During the third week of lactation, does receiving diet $\mathrm{L}$ exhibited a significantly higher weight loss than the control ones : $-208 \mathrm{~g} v \mathrm{~s}-66 \mathrm{~g}$.

Body stores at weaning were not different in does fed the experimental diets only during lactation. They were reduced when diet $L$ was offered before the first mating (trial 1).

Diet $\mathrm{L}$ led to a reduction in the live weight of young rabbits and in the litter weight at weaning. By contrast, mortality rate beween 0 and 29 days of young rabbits from does fed diet $\mathrm{L}$ only reached 8 p. 100 versus 14.4 p. 100 in young from does fed the control diet. The excess of calcium in diet $\mathbf{L}$ and its digestible energy deficiency are probably partly responsible for these results.

Key words : Doe rabbit, reproduction, mortality, lucerne, dietary balance. 


\section{Références bibliographiques}

Bachacou J., Masson J.P., Millier C., 1981. Le programme COVAR in : Manuel de la Programmathèque statistique Amance, 81, 459-480, I.N.R.A., éd. Paris.

Colin M., Arkhurst G., Lebas F., 1973. Effet de l'addition de méthionine au régime alimentaire sur les performances de croissance chez le lapin. Ann. Zootech., 22, 485-492.

Bombeke A., Okerman F., Mormans R., 1978. L'influence de la granulation à sec et à la vapeur des rations à teneurs différentes en énergie sur les résultats de production des lapins de chair. Revue de l'Agriculture, 31, 945-955.

Coudert P., Lebas F., 1985. Production et morbidité de lapines reproductrices. 1. Effets du rationnement alimentaire avant et pendant la première gestation. Ann. Zootech., 34, 31-48.

Jouglar J.-Y., 1983. Contribution à l'étude du syndrome «Parésie gastro-intestinale» de la lapine allaitante. Thèse de Doctorat $3^{\mathrm{e}}$ Cycle. Sciences et Techniques en Production Animale, I.N.P. Toulouse, 142 p.

Lang J., 1981. The nutrition of the commercial rabbit. Part 1. Physiology, serie digestibility and nutrient requirements. Nutr. Abst. Rev., 51 B, 197-225.

Lebas F., 1968. Mesure quantitative de la production laitière chez la lapine. Ann. Zootech., $17,169-182$.

Lebas F., 1977. Alimentazione delle riproduttrici. Coniglicoltura, 14 (12), 11-16.

LEBAS F., 1987. Influence de la taille de la portée et de la production laitière sur la quantité d'aliment ingérée par la lapine allaitante. Reprod. Nutr. Dévelop., 27 (sous presse).

Lebas F., Jouglar J.Y., 1984. Apports alimentaires de calcium et de phosphore chez la lapine reproductrice. Mémoire $3^{\circ}$ Congrès Mondial de Cuniculture, Rome, avril 1984, Vol. 1, 461-466.

Perret J.-P., Dorier A., Bacques C., 1977. Etude de l'évolution de la nature des triglycérides du lait pendant les trois premières semaines de lactation chez la lapine normale ou tarie précocement. Ann. Biol. Anim. Biochim. Biophys., 17, 153-163. 